\title{
Partial order based approach to synthesis of speed-independent circuits*
}

\author{
Alex Semenov, Alex Yakovlev \\ Department of Computing Science \\ University of Newcastle \\ Newcastle upon Tyne, NE1 7RU \\ England
}

\author{
Enric Pastor, Marco A. Peña, \\ Jordi Cortadella \\ Department of Computer Architecture \\ Universitat Politècnica de Catalunya \\ 08071 Barcelona, Spain
}

\author{
Luciano Lavagno \\ Dip. di Elettronica \\ Politecnico di Torino \\ C. Duca degli Abruzzi 24 \\ 10129 Torino, ITALY
}

\begin{abstract}
This paper introduces a novel technique for synthesis of speed-independent circuits from their Signal Transition Graph specifications. The new method uses partial order in the form of the STG-unfolding segment to derive the logic implementation using approximation techniques. It is based on a new notion of slice, which localises the behaviour of a particular signal instance in a structural fragment of the segment. The experimental results show the power of the approximation approach in comparison with the existing methods.
\end{abstract}

\section{Introduction}

There exists a variety of approaches to synthesis of speed-independent circuits from their Signal Transition Graph (STG) specifications. These approaches can be divided according to the library of elements used in implementations. For example, $[5,1]$ use a memory latch for each signal and a network of gates to drive it. Early methods, e.g. [2], assume that each signal is implemented as a single complex gate. Later techniques, e.g. $[16,6]$, attempt to decompose the complex gates preserving the speed-independence ${ }^{1}$ of the circuit.

Two primary approaches exist to date: State Graph (SG) based and structural methods. The first approach constructs a $S G$ and extracts subsets of states required for implementation. This method is used in such tools as SIS [15] and Assassin [18]. A recently developed tool Petrify (and ap-

"This work was supported in part by the EPSRC grant No. GR/J 52327, ESPRIT ACiD-WG Nr.21949, MURST project "VLSI architectures" and grant CICYT TIC95-0419. Collaboration between University of Newcastle and Universitat Politècnica de Catalunya was supported by British-Spanish joint research programme (Acciones Integradas) between the British Council and Spanish Ministry of Education and Science, grant numbers MDR(1996/97)1159 and UK HB1995-0203

${ }^{1}$ Speed-independent circuits are hazard-free for any delays attached to their gate outputs. proach) [3] uses Binary Decision Diagrams (BDDs) to represent the state space. The SG based methods suffer from the state explosion, i.e. the number of reachable states grows exponentially with the size of the specification.

The structural method of [10] uses State Machine (SM) decompositions to find an implementation avoiding the state exploration. Although it demonstrated impressive results, it is restricted to free-choice specifications.

Partial order techniques have also been applied in the synthesis process. Change Diagrams were introduced in [5] for synthesis of speed independent circuits from choice-free (no choice at all) specifications. More recent work in [9] used Petri Net (PN) unfoldings to derive logic functions. This work, however, is based on restoring the state space from the partial order and is therefore also prone to state explosion.

STG specifications can be verified efficiently using the STG-unfolding segment [13]. In a vast number of examples the segment can be constructed and verified where the construction of the $S G$ fails. Thus, after the verification is completed the segment can be used for deriving the logic functions of implementation.

This paper proposes a novel approach for the synthesis of speed independent circuits from the STG-unfolding segment of their specifications. It introduces a new notion of slice which helps to localise the behavioural information for signal instances within a structural fragment of the segment. An exact approach, producing implementations comparable with those of the $S G$ approach, is given. To overcome the state explosion problem, an approximation method based on temporal relations found in the segment is suggested. However, unlike [10], our approach uses local dependency information available for each instance of every signal transition. Only instances of signal transitions which are concurrent to a particular instance are considered for it. This gives a more accurate initial approximation and a more precise refinement. We illustrate our approach applying it to synthesis in three major implementation architectures. The scope 


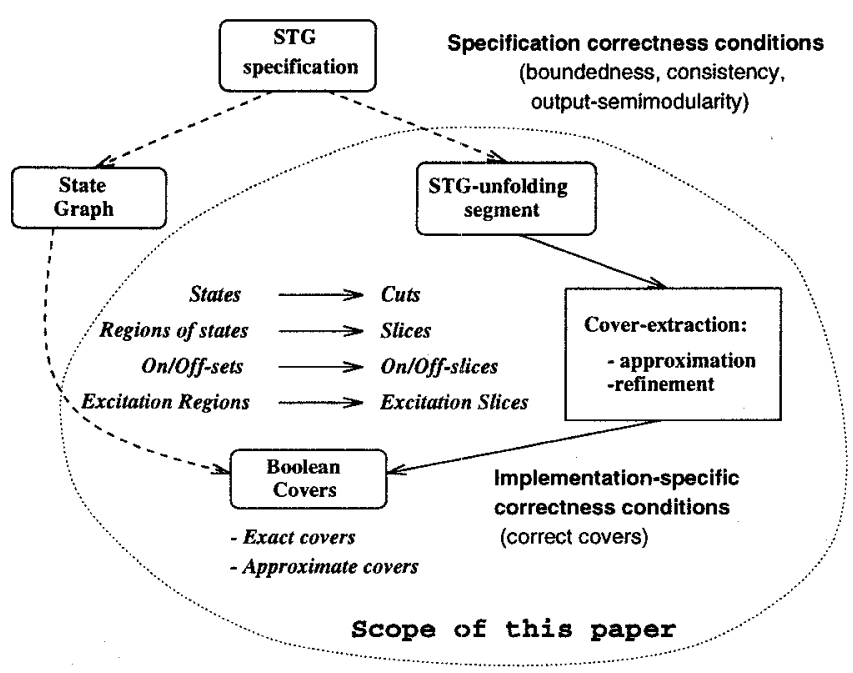

Figure 1. Overview of synthesis issues discussed in the paper.

of synthesis issues presented in this paper is summarised in Figure 1.

The paper is organised as follows. Section 2 describes the general approach and architectures for synthesis of the speed-independent circuits from their STG specifications. Section 3 introduces new notions required for synthesis from the STG-unfolding segment such as cuts and slices. Section 4 describes the ways for exact cover extraction from the STG-unfolding. Section 5 presents our main result the cover extraction method based on approximation and refinement. The results of experiments, showing performance and comparing the new method with the existing ones are in Section 6.

\section{Speed-Independent Circuit Implementation}

This section introduces the basic concepts required to develop the synthesis of speed-independent circuits. Further reading on Petri nets, Signal Transition Graphs, and logic synthesis can be obtained at $[11,12,2]$.

\subsection{Basic Synthesis Concepts}

A Petri net (PN) is a 4-tuple $\left\langle P, T, F, m_{o}\right\rangle$, with sets of places $P$, transitions $T$, flow relation $F$ and initial marking $m_{o}$. A marking $m$ is represented with a number of tokens $m(p)$ in each place $p \in P$. A Signal Transition Graph (STG) $[12,2]$ is a triple $\langle N, A, L\rangle$ where $N$ is a PN, $A$ is a set of signals, and $L: T \rightarrow\{+,-\} \times A$ is a labelling function that assigns a signal switch to each transition in $T$. An STG is a special case of labelled PN, particularly useful to describe low level asynchronous circuits. The set of transitions represents up $\left(+a_{i}\right)$ and down $\left(-a_{i}\right)$ switching activity. Notation $* a_{i}$ is used to indicate a signal transition regardless of the direction of the change. Given an element $x \in T \cup P$, its immediate predecessors and successors sets are denoted $\bullet x$ and $x \bullet$ respectively.

An STG is called $k$-bounded iff the number of tokens in any place $p_{i} \in P$ at any reachable marking does not exceed $k$. Boundedness guarantees that an STG can be implemented using a finite number of memory elements. An STG is called output semi-modular iff no output signal transition $* a_{i}$ excited at any reachable marking can be disabled by transition of another signal $* a_{j}$ (also known as output signal persistency [5]). If an STG is output semimodular, then can be implemented without producing unspecified changes of the output signals; that is, without introducing hazards [5].

To obtain an implementation for an STG (e.g. in Figure 2(a)), the SG (Figure 2(b)) is derived by constructing the reachability graph for the STG (representing all reachable states), and then assigning a binary code $v_{i}$ to each state $s_{i}$. The binary codes must be assigned consistently. An STG is called consistent if its SG has a consistent state assignment. The Complete State Coding (CSC) condition introduced in [2] requires any two states with equal binary codes to have the same set of excited output signals. If for some signal $a_{i}$ this requirement is not satisfied, then it is impossible to extract the boolean function for its implementation. It was shown in [2] that STGs satisfying CSC are implementableas speed-independent circuits.

An STG model satisfying the general specification correctness conditions (boundedness, consistency, output semi-modularity) and producing an SG with CSC, gives rise to truth tables. However, the process of obtaining a truth table depends on a particular implementation architecture chosen for each signal.

The following three architecture types are commonly considered for the synthesis of speed-independent circuits:

1. Atomic complex gate per signal (ACGpS) implementation;

2. Atomic complex gate per excitation function (ACG$\mathrm{pEF}$ ) implementation;

\section{Atomic complex gate per excitation region (ACGpER) implementation.}

Implementations are obtained by building cover functions which are then directly associated with the elements in the circuit. A boolean function covers a state $s_{j}$ if the function evaluates to TRUE when the variables have their values equal to the signals at the binary code $v_{j}$. A function covering a set of states is called a cover function (or simply cover). Each term of the cover is called cube as it may cover several states in the state space.

An exact cover for a set of states $\left\{s_{i}\right\}$ can be obtained directly from the set of binary codes $v_{j}$, but it will require an 


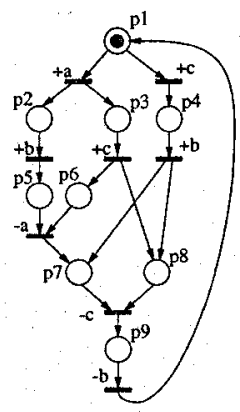

(a)

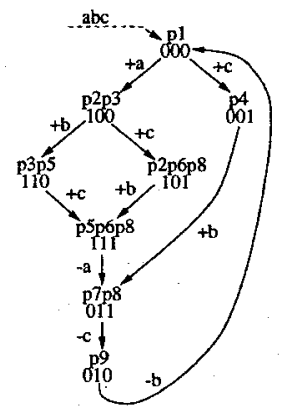

(b)

\section{Figure 2. An example of an STG and its cor- responding SG.}

explicit enumeration of all the states. Approximated covers can be generated using some structural information from the STG. However, implementations created by using approximated covers require additional checking for correctness.

The notion of correctness, applied at the implementation stage, is concerned with the requirement of hazardfreedom with respect to the gates of the synthesized circuit. Architecture-specific correctness criteria put into correspondence the sets of states $s_{i}$ and their cover functions.

In order to further demonstrate our unfolding-based method we chose relatively simple cover correctness criteria which, however, guarantee existence of the implementation in all three architectures for a CSC-compliant STG.

\subsection{Atomic Complex Gate per Signal}

This architecture for speed-independent circuits was studied in $[2,12]$. The circuit is implemented as a network of atomic gates, each one implementing one output signal. The boolean function for each gate can be represented as Sum-Of-Products (SOP). Such a gate is illustrated in Figure 3(a). Each atomic gate contains a combinational part, and a possibly sequential part implemented as an internal feedback where internal delays are negligible.

Two (mutually complementary) subsets of the reachable states are distinguished in the SG for every signal $a_{i}$, onset $O n\left(a_{i}\right)$ and off-set $O f f\left(a_{i}\right)$, which include all states in which the value of the output signal $a_{i}$ is implied to be TRUE and FALSE, respectively. The remaining (unreachable) subset of combinations of the boolean values of signals forms the Don't care set (DC-set).

The implementation is derived by finding the on-set (the off-set may be chosen if it leads to a simpler circuit). The cover $\mathcal{C}$ for the implementation is obtained from the terms assigned to the states included into the on-set. The DC-set can be used for optimising the size of $\mathcal{C}$ applying standard minimisation tools such as Espresso [15].

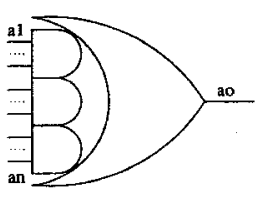

(a)

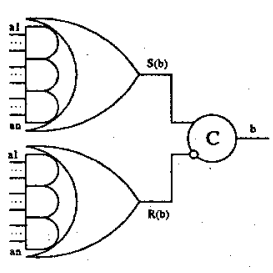

(b)

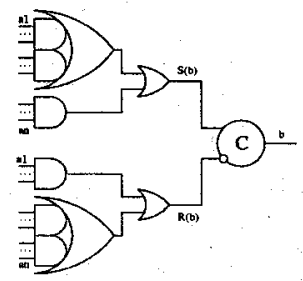

\section{Figure 3. Illustration of implementation archi- tectures.}

Obtaining exact covers usually means that all states in the on- or off-set must be known. An approximation algorithm produces approximated covers of the on- and off-sets. Therefore, in this implementation architecture, the covers of on- and off-sets (either exact or approximated) must satisfy the following condition:

Definition 2.1 Given an output signal $a_{i}$, two covers $\mathcal{C}_{\text {On }}\left(a_{i}\right)$ and $\mathcal{C}_{\text {Off }}\left(a_{i}\right)$ are said to be correct iff $\mathcal{C}_{\text {On }}\left(a_{i}\right)$ and $\mathcal{C}_{\text {off }}\left(a_{i}\right)$ cover $O n\left(a_{i}\right)$ and $O f f\left(a_{i}\right)$ respectively and $\mathcal{C}_{\text {On }}\left(a_{i}\right) \cdot \mathcal{C}_{\text {Off }}\left(a_{i}\right) \subseteq$ DC-set.

A correct cover may become TRUE when its variables take values corresponding to the combinations belonging to the DC-set.

\subsection{Atomic Complex Gate per Excitation Function}

The ACGpEF architecture was suggested and studied extensively in a number of papers, e.g. [6, 1]. It assumes that a separate memory element is used to produce an output signal. The Set and Reset excitation functions for this memory element are implemented as atomic complex gates. Depending on which memory element is used, the implementations are divided into: i) Standard $C$-element implementation, which uses Muller C-element as the memory element (shown in Figure 3(b)), and ii) Standard RS-latch implementation, where an RS-latch is used.

A Generalised Excitation Region (GER) of $* a_{i}$, denoted as $G E R\left(* a_{i}\right)$, is defined as a set of states of SG which has an arc coming out of them which is labelled with $* a_{i}$. For each signal there exist two GERs found for all positive $\left(G E R\left(+a_{i}\right)\right)$ and negative $\left(G E R\left(-a_{i}\right)\right)$ transitions. GERs represent all states in which signal $a_{i}$ is excited. In an output semi-modular STG, an output signal $a_{i}$ cannot be disabled by firing any other signal. Thus $G E R\left(+a_{i}\right)$ and $G E R\left(-a_{i}\right)$ can only be left through the firing of $+a_{i}$ and $-a_{i}$, respectively.

An implementation is built by finding covers $\mathcal{C}_{S}$ and $\mathcal{C}_{R}$ as set and reset functions, obtained from the terms corresponding to the states in $G E R\left(+a_{i}\right)$ and $G E R\left(-a_{i}\right)$. It is 
possible to show the existence of an implementation in this architecture for any STG satisfying the CSC condition [2].

The cover correctness condition in this architecture is as follows:

Definition 2.2 A set (reset) cover $\mathcal{C}_{S}\left(a_{i}\right)\left(\mathcal{C}_{R}\left(a_{i}\right)\right)$ is said to be correct if the only reachable states covered by $\mathcal{C}_{S}\left(a_{i}\right)$ $\left(\mathcal{C}_{R}\left(a_{i}\right)\right)$ belong to $G E R\left(+a_{i}\right)\left(G E R\left(-a_{i}\right)\right)$ and the cover covers all states in this GER.

Note that this condition does not restrict the correct cover as the one which covers GER exactly. The latter is a special case of the correct cover. An approximated cover may also include states from the DC-set. An implementation of an STG with CSC by means of exact GER covers always exists as they satisfy this correctness condition.

\subsection{Atomic Complex Gate per Excitation Region}

Signals in this architecture are created using networks of atomic complex gates to implement set and reset functions of the memory element. As a result, smaller complex gates are used which are then connected to an OR-gate whose output is in turn fed into the memory element. The basic structure of this architecture is shown in Figure 3(c). Similar to the previous architecture, the memory element can be a Muller C-element or an RS-latch.

To implement a set (reset) function, excitation regions of a signal are defined. An excitation region (ER) of a signal $a_{i}$, denoted as $E R\left(* a_{i}\right)$, is a maximal set of connected states of SG which have an outgoing arc labelled with $* a_{i}$. Obviously, if there are several $E R_{j}\left(* a_{i}\right)$ for one signal $a_{i}$, then $G E R\left(* a_{i}\right)=\cup E R_{j}\left(* a_{i}\right)$.

The correctness condition for this architecture is as follows:

Definition 2.3 A set of covers for set (reset) function of signal $a_{i}, \mathcal{C}_{S}^{1}\left(a_{i}\right), \ldots, \mathcal{C}_{S}^{n}\left(a_{i}\right)\left(\mathcal{C}_{R}^{1}\left(a_{i}\right), \ldots, \mathcal{C}_{R}^{m}\left(a_{i}\right)\right)$, is said to be correct if each $E R_{j}\left(+a_{i}\right)\left(E R_{j}\left(-a_{i}\right)\right)$ is covered by its corresponding $\mathcal{C}_{S}^{j}\left(a_{i}\right)\left(\mathcal{C}_{R}^{j}\left(a_{i}\right)\right)$ and the only reachable states covered by $\mathcal{C}_{S}^{j}\left(a_{i}\right)\left(\mathcal{C}_{R}^{j}\left(a_{i}\right)\right)$ belong to $E R_{j}\left(+a_{i}\right)$ $\left(E R_{j}\left(-a_{i}\right)\right.$.

Since the union of all unconnected ERs is a GER, then all states in GER will be covered. Similar to the previous architecture, the covers need not cover their ERs exactly. Exact covers are a special case which always satisfies this condition. In an extreme case, when the set cover consists of only one cover the OR-gate, supposed to merge the outputs of gates implementing ER covers, becomes redundant.

\section{Slices in STG-unfolding segment}

This section introduces definitions needed for our proposed synthesis method. First, the concept of an STG- unfolding segment is outlined. Then, we introduce the notions of cuts and slices which allows us to localise the behavioural information of a particular signal instance.

\subsection{STG-unfolding segment}

Analysis of STGs using STG-unfolding segment was studied elsewhere [13]. An STG-unfolding segment for an STG $G$, is $G^{\prime}=\left\langle T^{\prime}, P^{\prime}, F^{\prime}, \Lambda^{\prime}\right\rangle$ where $T^{\prime}, P^{\prime}$ and $F^{\prime}$ are sets of transitions, places and the flow relation, respectively; and $\Lambda^{\prime}$ is a labelling function which labels each element of $G^{\prime}$ as an instance of elements of $G . G^{\prime}$ is a partial order obtained from an STG $G$ by the process of its unfolding. In the unfolding the relations of conflict, concurrency and precedence are used to decide where to instantiate the next element. These relations are constructed during the unfolding process from the basic flow relation $F^{\prime}$, built from the flow relation $F$ in the original STG. More formally, a transitively closed (w.r.t. $F^{\prime}$, which defines immediate predecessors of a place or transition instance) set of unfolding elements for an instance $x^{\prime}$ is called the history of $x^{\prime}$. For any pair of instances $x_{1}^{\prime}, x_{2}^{\prime} \in P^{\prime} \cup T^{\prime}$ in the unfolding three relations are defined:

- Precedence (or Sequence), denoted as $x_{1}^{\prime} \preceq x_{2}^{\prime}$, iff $x_{1}^{\prime}$ belongs to the history of $x_{2}^{\prime}$.

- Conflict, denoted as $x_{1}^{\prime} \# x_{2}^{\prime}$, iff there exist two distinct transitions $t_{1}^{\prime}$ and $t_{2}^{\prime}$ in the histories of $x_{1}^{\prime}$ and $x_{2}^{\prime}$, respectively, such that $\bullet t_{1}^{\prime} \cap \bullet t_{2}^{\prime} \neq \emptyset$.

- Concurrency, denoted as $x_{1}^{\prime} \| x_{2}^{\prime}$, iff $x_{1}^{\prime}$ and $x_{2}^{\prime}$ are neither in conflict nor in sequence.

In contrast to PN-unfolding [8], the STG-unfolding takes into account specific signal interpretation of PN transitions and keeps track of the binary codes reached by transition firing. However, it still examines only a subset of all reachable states of $G$ and thus is more efficient than SG exhaustive analysis for a vast number of examples.

The minimal set (min-set) of transitions needed to fire $t^{\prime}$ in the STG-unfolding segment, is called the local configuration of $t^{\prime}$ and is denoted as $\left\lceil t^{\prime}\right\rceil$. A set of place instances reached by firing all transitions in $\left[t^{\prime}\right]$ is called the postset of $\left\lceil t^{\prime}\right\rceil$ and is as denoted $\left\lceil t^{\prime}\right\rceil \bullet$. Mapping a postset onto places of the STG gives a marking of the original STG. Any nonconflicting and transitively closed, w.r.t. the precedence relation, set of transitions of $T^{\prime}$ is called configuration.

Each instance $t^{\prime}$ of the STG-unfolding segment has a binary code $\xi_{\left[t^{\prime}\right\rceil}$ which is reached by firing transitions in $\left\lceil t^{\prime}\right\rceil$. The postset $C \bullet$ and binary code $\xi_{C}$ corresponding to a configuration $C$ are calculated from $\left\lceil t^{\prime}\right\rceil \bullet$ and $\xi_{\left[t^{\prime}\right\rceil}$ of transitions comprising it. It was shown in [13] that all states of the SG are represented in the STG-unfolding segment as postsets of some configuration. 
Note that the process of constructing the STG-unfolding segment (which is a finite object for a bounded PN) is terminated at the transition instances, called cut-off points, whose mapped final state $\left\lceil t^{\prime}\right\rceil$ is equal to the final state of some other instance already put into the segment. There exist several definitions of the cut-off condition [13,4], different in their attempts to minimize the size of the truncated PN (or STG) unfolding necessary to fully represent the SG.

A special transition, called the initial transition, is introduced in the unfolding to represent the initial state of the STG. This transition, denoted as $\perp$, has a postset which maps onto the initial marking $m_{0}$ and has an assigned binary code $\xi_{[\perp]}$ equal to that of the initial state $v_{0}$ of the STG.

It was demonstrated in [13] that an STG-unfolding segment can only be constructed for a bounded and consistent STG specification. The last general correctness criterion, output semi-modularity, can be checked on the STGunfolding segment in linear time.

\subsection{Cuts of STG-unfolding segment}

To represent a state of the SG we define a cut.

Definition 3.1 $A$ cut of an STG-unfolding segment is a maximal set of mutually concurrent places $p^{\prime} \in P^{\prime}$.

Each cut $c$ thus represents some reachable marking of the original STG. A sequence relation is defined between two cuts $\mathrm{C}_{1} \preceq \mathrm{C}_{2}$ if $\forall p_{i}^{\prime} \in \mathrm{C}_{2}, \exists p_{j}^{\prime} \in \mathrm{C}_{1}: p_{j}^{\prime} \preceq p_{i}^{\prime}$. Since a cut $C$ represents a marking, then there exists a configuration $C$ in the STG-unfolding segment whose postset is equal to $C$, i.e. $C \bullet=$ c.

For each instance $t^{\prime}$ four types of cuts can be found. First two types are minimal cuts, i.e. cuts reached by some minimal run of the STG. The other two types of cuts are maximal cuts, i.e. cuts from which the system cannot make any further progress unless some condition is violated.

Definition 3.2 A cut $c_{e}^{\min }\left(t_{k}^{\prime}\right)$ is called a minimal excitation cut of $t_{k}^{\prime}$ iff $\bullet t_{k}^{\prime} \subseteq \mathrm{C}_{e}^{m i n}\left(t_{k}^{\prime}\right)$ and $\forall t_{j}^{\prime}, t_{j}^{\prime} \| t_{k}^{\prime}$ : $\left(t_{j}^{\prime} \bullet\right) \cap \mathrm{c}_{e}^{\min }\left(t_{k}^{\prime}\right)=\emptyset$.

Minimal excitation cut represents the state at which $t_{k}^{\prime}$ becomes first enabled.

Definition 3.3 A cut $\mathrm{C}_{s}^{\text {min }}\left(t_{k}^{\prime}\right)$ is called a minimal stable cut of $t_{k}^{\prime}$ iff $\left.t_{k}^{\prime} \bullet \subseteq C_{s}^{m i n}\left(t_{k}^{\prime}\right)\right)$ and $\forall t_{j}^{\prime}, t_{j}^{\prime} \| t_{k}^{\prime}:\left(t_{j}^{\prime} \bullet\right) \cap$ $c_{s}^{m i n}\left(t_{k}^{\prime}\right)=\emptyset$.

Minimal stable cut represents a state which is reached by firing of $t_{k}^{\prime}$ enabled after firing of transitions in its $\left\lceil t_{k}^{\prime}\right\rceil$. Minimal excitation and stable cuts for one instance $t_{k}^{\prime}$ are two adjacent cuts which are separated by $t_{k}^{\prime}$.

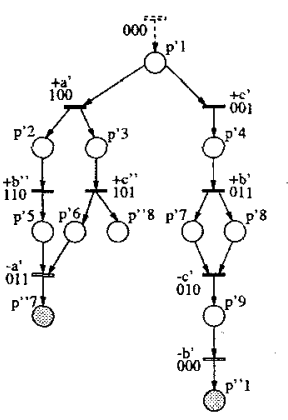

(a)

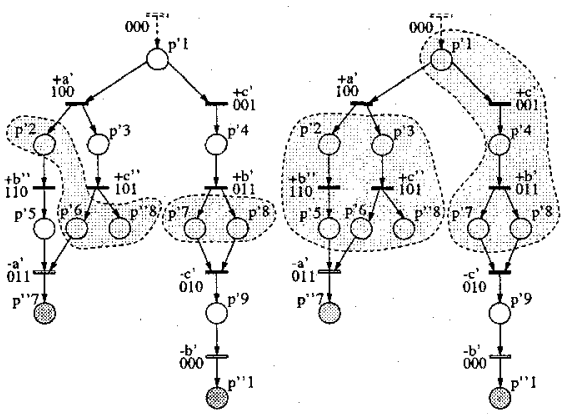

(b)

(c)

\section{Figure 4. An example of an STG-unfolding segment and illustration of slices and cuts.}

Definition 3.4 A cut $\mathrm{C}_{e}^{\text {max }}\left(t_{k}^{\prime}\right)$ is called maximal excitation cut of $t_{k}^{\prime}$ iff $\nexists \mathrm{c}_{j}, \bullet t_{k}^{\prime} \subseteq \mathrm{c}_{j}: \mathrm{c}_{e}^{\max }\left(t_{k}^{\prime}\right) \prec \mathrm{c}_{j}$.

Maximal excitation cuts represent states from which no advancement can be made unless $t_{k}^{\prime}$ is fired.

Definition 3.5 A cut $c_{s}^{\max }\left(t_{k}^{\prime}\right)$ is called maximal stable cut of $t_{k}^{\prime}$ iff $c_{s}^{\min }\left(t_{k}^{\prime}\right) \preceq c_{s}^{\max }\left(t_{k}^{\prime}\right)$ and firing of any transition $t_{j}$ enabled at the marking corresponding to $\mathrm{C}_{s}^{\max }\left(t_{k}^{\prime}\right)$ reaches a marking enabling the next change of $a_{i}$ labelling $t_{k}^{s}$.

A special case is a maximal stable cut for the initial transition $\perp$. It is found w.r.t. a particular signal $a_{i}$ as if $\perp$ was labelled with an appropriate change of $a_{i}$.

Finally, note that each instance $t_{k}^{\prime}$ of the STG-unfolding segment uniquely identifies $C_{e}^{\text {min }}\left(t_{k}^{\prime}\right)$ and $C_{s}^{\min }\left(t_{k}^{\prime}\right)$ and the sets of $\mathrm{C}_{e}^{\max }\left(t_{k}^{\prime}\right)$ and $\mathrm{c}_{s}^{\max }\left(t_{k}^{\prime}\right)$.

Example. Several cuts are illustrated in Figure 4. Consider a cut $\mathrm{c}=\left(p_{7}^{\prime}, p_{8}^{\prime}\right)$ in Figure $4(b)$. This cut is a minimal excitation cut for the transition $-c^{\prime}$ and is a minimal stable cut for $+b^{\prime}$. Another cut, $\mathrm{c}=\left(p_{2}^{\prime}, p_{6}^{\prime}, p_{8}^{\prime \prime}\right)$ is a maximal stable cut for transition instance $+a^{\prime}$. At the same time this is a maximal excitation cut for the instance $+b^{\prime \prime}$.

\subsection{Slices of STG-unfolding segment}

To represent a connected set of states we introduce the notion of slice.

Definition 3.6 A slice of an STG-unfolding segment is a set of cuts $\mathcal{S}$ defined by a pair $\left\langle\mathrm{C}^{\min }, \mathrm{C}^{\max }\right\rangle$ where $\mathrm{c}^{\min }$ is min-cut of the slice, and $C^{\max }$ is a set of max-cuts such that $\forall c_{i} \in \mathcal{S}$

1. $c^{\min } \preceq \mathrm{c}_{i}$ and $\exists c_{j} \in \mathrm{C}^{\max }: \mathrm{c}_{i} \preceq \mathrm{c}_{j}$, and

2. no two cuts in the set of max-cuts in $C^{\max }$ are mutually ordered by the sequence relation. 
Every cut in between the min-cut and a max-cut is encapsulated in the slice $\mathcal{S}$. Furthermore, for any two cuts $\mathrm{C}_{i}$ and $c_{j}$, if $c_{i} \prec c_{j}$, then all cuts between $c_{i}$ and $c_{j}$ are also encapsulated by $\mathcal{S}$. Since each cut represents some state in the $\mathrm{SG}$, for any two states $s_{i}$ and $s_{j}$ represented as sequential cuts in $\mathcal{S}$, all states on any path from $s_{i}$ to $s_{j}$ are also represented as cuts encapsulated into $\mathcal{S}$. The number of cuts in the set of max-cuts corresponds to the number of configurations which include configuration producing the min-cut. The elements of the STG-unfolding segment, i.e. places, transitions and arcs, bounded by the instances in min-cut and max-cuts are said to belong to the slice.

Example. Slice $\mathcal{S}_{1}=\left\langle\left(p_{1}^{\prime}\right),\left\{\left(p_{7}^{\prime}, p_{8}^{\prime}\right)\right\}\right\rangle$ in Figure $4(c)$ encapsulates cut $\mathrm{C}=\left(p_{4}^{\prime}\right)$. Another slice $\mathcal{S}_{2}$ is defined between a min-cut $\left(p_{2}^{\prime}, p_{3}^{\prime}\right)$ and a set of max-cuts $\left\{\left(p_{5}^{\prime}, p_{6}^{\prime}, p_{8}^{\prime \prime}\right)\right\}$ and includes all cuts between them. It is also possible to define a slice between $\left(p_{2}^{\prime}, p_{3}^{\prime}\right)$ and $\left\{\left(p_{2}^{\prime}, p_{6}^{\prime}, p_{8}^{\prime \prime}\right),\left(p_{3}^{\prime}, p_{5}^{\prime}\right)\right\}$. In this case the slice will include all cuts but the one enabling $-a^{\prime}$.

Each cut is produced by some configuration of the STGunfolding segment. Thus the binary codes of the SG states represented by cuts encapsulated in a particular slice can be recovered by examining its cuts.

\section{Obtaining exact covers from STG-unfolding segment}

To implement an output signal in any architecture, specific subsets of states from the $S G$ are required. A slice represents a set of states of the SG in form of the STGunfolding segment $G^{\prime}$. Therefore, the problem is posed as finding a partitioning of the segment into slices representing appropriate states. To define each slice we need to identify its min-cut and a set of max-cuts. Consider finding these for each architecture.

\subsection{Atomic Complex Gate per Signal}

As it was shown in [14], the set of slices representing states in the on-set (off-set) can be identified on the STGunfolding segment using the instances of signal transitions $* a_{i}^{\prime}$ and an initial transition $\perp$. The initial transition is used when the signal $a_{i}$ is at " 1 " (" 0 ") in the initial state of the STG. The slices are bounded by the minimal excitation slice and a set of maximal stable cuts uniquely determined by each instance. Thus the On-set and Off-set partitioning in an STG-unfolding segment are defined as follows.

Definition 4.1 A set of slices $\mathbb{S}_{O n}\left(\mathbb{S}_{O f f}\right)$ in an STGunfolding segment $G^{\prime}$ is called On-set (Off-set) partitioning of $G^{\prime}$ w.r.t. a signal $a_{i}$ iff for each instance $+a_{i}^{\prime}$ $\left(-a_{i}^{\prime}\right)$ its corresponding slice $\mathcal{S}_{O n}^{j}\left(+a_{i}^{\prime}\right)\left(\mathcal{S}_{O f f}^{j}\left(-a_{i}^{\prime}\right)\right)$ is

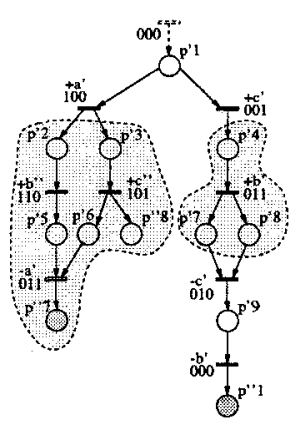

(a)

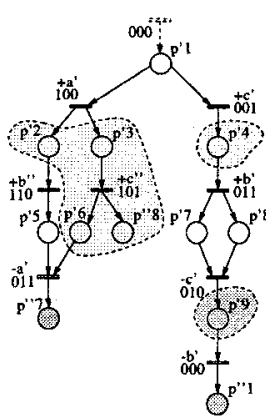

(b)

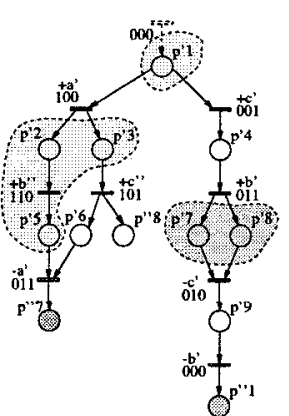

(c)
Figure 5. Illustration of synthesis from the STG-unfolding segment.

defined with $\mathrm{C}_{e}^{\min }\left(+a_{i}^{\prime}\right)\left(\mathrm{C}_{e}^{\min }\left(-a_{i}^{\prime}\right)\right)$ as its min-cut and $\mathrm{C}_{s}^{\max }\left(+a_{i}^{\prime}\right)\left(\mathrm{C}_{s}^{\max }\left(-a_{i}^{\prime}\right)\right)$ as a set of its max-cuts.

If signal $a_{i}$ is at " 1 " (" 0 ") in the initial state of the STG, then the set of slices $\mathbb{S}_{O n}\left(\mathbb{S}_{O f f}\right)$ also includes slice defined with $C_{s}^{\text {min }}(\perp)$ as a min-cut and $C_{s}^{\max }(\perp)$ as its set of max-cuts.

The on-set cover is obtained from the On-set partitioning by extracting the binary codes corresponding to the cuts encapsulated by the slices:

$$
\mathcal{C}_{\text {On }}=\sum \mathcal{C}_{\text {On }}\left(+a_{i}^{\prime}\right)
$$

where $\mathcal{C}_{O n}=\bigcup \xi_{C_{j}}, C_{j} \bullet=\mathrm{c}_{j} \in \mathcal{S}_{O n}\left(+a_{i}^{\prime}\right)\left(\mathcal{S}_{O n}(\perp)\right.$ is also used, if required). The on-set cover is found as the union of all covers found for the slices of on-set partitioning. Each slice cover is obtained by taking the union of all minterms corresponding to the binary codes of configurations $C_{j}$ whose postset is a cut $c_{j}$ encapsulated by $\mathcal{S}_{O n}\left(+a_{i}^{\prime}\right)$. Slice $\mathcal{S}_{O n}\left(+a_{i}^{\prime}\right)$ is called on-set slice.

Example. Consider synthesising signal b from an example in Figure 4. The on-set partitioning of the STG-unfolding segment is shown in Figure 5(a). There are two instances $+b^{\prime}$ and $+b^{\prime \prime}$. Thus there are two slices $\mathcal{S}_{O n}^{1}\left(+b^{\prime}\right)=\left\langle\left(p_{4}^{\prime}\right),\left\{\left(p_{7}^{\prime}, p_{8}^{\prime}\right)\right\}\right\rangle$ and $\mathcal{S}_{O n}^{2}\left(+b^{\prime \prime}\right)=$ $\left\langle\left(p_{2}^{\prime}, p_{3}^{\prime}\right),\left\{\left(p_{7}^{\prime \prime}, p_{8}^{\prime \prime}\right)\right\}\right\rangle$ representing states from the on-set. The on-set cover is obtained from slices as $\mathcal{C}_{O n}=$ $\{100,101,110,111,011\} \cup\{001,011\}$ which after standard boolean transformation gives $\mathcal{C}_{O n}=\{1--,--1\}=$ $a+c$ :

\subsection{Atomic Complex Gate per Excitation Function}

A set of slices representing the GERs of $* a_{i}$ is found in the STG-unfolding segment using the instances of this signal $* a_{i}^{\prime}[14]$. Any cut representing a state belonging to 
GER of $* a_{i}$ is encapsulated between the minimal excitation cut and the set of maximal excitation cuts, uniquely identified by one of its instances $* a_{i}^{\prime}$. By finding all slices representing states in GER we obtain a cover satisfying the correctness condition for this architecture.

Definition 4.2 A set of slices $\mathbb{S}_{S}\left(a_{i}\right)\left(\mathbb{S}_{R}\left(a_{i}\right)\right)$ in an STGunfolding segment $G^{\prime}$ is called Set (Reset) partitioning of the STG-unfolding segment $G^{\prime}$ w.r.t. signal $a_{i}$ iff for each instance $+a_{i}^{\prime}\left(-a_{i}^{\prime}\right)$ its corresponding slice $\mathcal{S}_{e}\left(+a_{i}^{\prime}\right)$ $\left(\mathcal{S}_{e}\left(-a_{i}^{\prime}\right)\right)$ is defined with $\mathrm{c}_{e}^{\text {min }}\left(+a_{i}^{\prime}\right)\left(\mathrm{c}_{e}^{\text {min }}\left(-a_{i}^{\prime}\right)\right)$ as its min-cut and $C_{e}^{\max }\left(+a_{i}^{\prime}\right)\left(C_{e}^{\max }\left(-a_{i}^{\prime}\right)\right)$ as a set of its maxcuts.

Each slice $\mathcal{S}_{e}\left(* a_{i}^{\prime}\right)$ is called excitation slice of $* a_{i}^{\prime}$. Similar to the previous architecture, the cover for set function is obtained from the binary codes corresponding to the cuts encapsulated by slices:

$$
\mathcal{C}_{S}\left(a_{i}\right)=\sum \mathcal{C}_{S}\left(+a_{i}^{\prime}\right)
$$

where $\mathcal{C}_{S}\left(+a_{i}^{\prime}\right)=\bigcup_{j} \xi_{C_{j}}, C_{j} \bullet=c_{j}: c_{j} \in \mathcal{S}_{e}\left(+a_{i}^{\prime}\right)$. As in the previous architecture, the covers are found by taking the union of minterms corresponding to the binary codes of configurations whose postsets are the cuts encapsulated by slices $\mathcal{S}_{\mathrm{e}}^{k}\left(+a_{i}^{\prime}\right)$. The reset function is obtained similarly using the $-a_{i}^{\prime}$ instances.

Example. Consider the synthesis of signal $b$ from $e x-$ ample in Figure 5(b). Two excitation slices are found as $\mathcal{S}_{e}\left(+b^{\prime}\right)=\left\langle\left(p_{2}^{\prime}, p_{3}^{\prime}\right),\left\{\left(p_{2}^{\prime}, p_{6}^{\prime}, p_{8}^{\prime \prime}\right)\right\}\right\rangle$ and $\mathcal{S}_{e}\left(+b^{\prime \prime}\right)=\left\langle\left(p_{4}^{\prime}\right),\left\{\left(p_{4}^{\prime}\right)\right\}\right\rangle$ for GER of $+b$ and $\mathcal{S}_{e}\left(-b^{\prime}\right)=$ $\left\langle\left(p_{9}^{\prime}\right),\left\{\left(p_{9}^{\prime}\right)\right\}\right\rangle$ for $-b$. After recovering the binary codes the covers for set and reset functions are: $\mathcal{C}_{S}=\{100,101\} \cup$ $\{001\}=\{10-,-01\}=a \bar{b}+\bar{b} c$ and $\mathcal{C}_{R}=\{010\}=\bar{a} b \bar{c}$.

\subsection{Atomic Complex Gate per Excitation Region}

The partitioning for this architecture is the same as for the Atomic Complex Gate per Excitation Function. The interpretation of the covers found for each slice is, however, different. Only covers whose intersection is non-empty represent one connected ER. Thus, after the covers for all excitation slices $\mathcal{S}_{e}\left(* a_{i}^{\prime}\right)$ are found the final implementation is obtained as a set of covers [14] corresponding to each connected ER:

$$
\text { for each } E R_{k}\left(* a_{i}\right): \mathcal{C}_{S}^{k}\left(* a_{i}\right)=\sum \mathcal{C}_{e}\left(* a_{i}^{\prime}\right)
$$

where $\forall \mathcal{C}_{e}\left(* a_{i}^{\prime}\right), \mathcal{C}_{e}\left(* a_{i}^{\prime \prime}\right)$ iff $\mathcal{C}_{e}\left(* a_{i}^{\prime}\right) \in \mathcal{C}_{S}^{k}\left(* a_{i}\right)$ and $\mathcal{C}_{e}\left(* a_{i}^{\prime}\right) \cdot \mathcal{C}_{e}\left(* a_{i}^{\prime \prime}\right) \neq \emptyset$ then $\mathcal{C}_{e}\left(* a_{i}^{\prime \prime}\right) \in \mathcal{C}_{S}^{k}\left(* a_{i}\right)$.

A fake conflict [7] situation may result in two slices with non-intersecting slices although the they represent one ER.
This situation is detected during STG verification and can be avoided by forcing the union of such covers, although we do not discuss it in detail here.

Example. Consider implementation of signal $c$ (Figure 5(c)). There are also two excitation slices for $+c: \quad \mathcal{S}_{e}\left(+c^{\prime}\right)=\left\langle\left(p_{1}^{\prime}\right),\left\{\left(p_{1}^{\prime}\right)\right\}\right\rangle$ and $\mathcal{S}_{e}\left(+c^{\prime \prime}\right)=$ $\left\langle\left(p_{2}^{\prime}, p_{3}^{\prime}\right),\left\{\left(p_{3}^{\prime}, p_{5}^{\prime}\right)\right\}\right\rangle$. However, $+c^{\prime}$ and $+c^{\prime \prime}$ are in fake conflict and these two slices represent one ER which is covered by $\mathcal{C}_{S}(c)=\bar{b} \bar{c}+a \bar{c}$. This, along with the reset cover $\mathcal{C}_{R}(c)=\bar{a} b c$ obtained from the excitation slice of $-c^{\prime}$, completes the implementation in this architecture.

\section{Deriving covers from STG-unfolding seg- ment efficiently}

The synthesis procedure described in the previous Section may suffer from exponential explosion of states. Recall that the correctness criteria for each architecture allow the implementing cover to be greater than the exact cover. This can be exploited in an approximation method for covering the desired slices (on-, off- and excitation slices).

\subsection{Strategies for deriving the covers}

There are two specific sets of reachable states for each cover $\mathcal{C}$ satisfying the correctness requirement:

- Positive set, denoted as $\mathcal{P}$-set, which is a set of states which $\mathcal{C}$ must cover; and

- Negative set, denoted as $\mathcal{N}$-set, which is a set of states which $\mathcal{C}$ must not cover.

The choice of the $\mathcal{P}$-set comes from the cover correctness conditions. $\mathcal{P}$-set is chosen to be the on-set for $\mathrm{ACGpS}$ and $G E R\left(+a_{i}\right)$ for ACGpEF and ACGpER. The $\mathcal{N}$-set is taken as the rest of reachable states. The generalised quiescent region of $+a_{i}\left(-a_{i}\right)$, denoted $G Q R\left(+a_{i}\right)$ $\left(G Q R\left(-a_{i}\right)\right)$, is a set of states at which signal $a_{i}$ is stable at " 1 " (" 0 "). Then the $\mathcal{N}$-set for the set (reset) function in ACGpEF and ACGpER implementations is chosen as $G Q R\left(+a_{i}\right) \cup G E R\left(-a_{i}\right) \cup G Q R\left(-a_{i}\right)\left(G Q R\left(-a_{i}\right) \cup\right.$ $\left.G E R\left(+a_{i}\right) \cup G Q R\left(+a_{i}\right)\right)$.

The cover is obtained by finding the partitioning representing the $\mathcal{P}$-set. However, the $\mathcal{N}$-set also corresponds to some slices. The interpretation of both sets and the covers and slices in the STG-unfolding segment suggests (at least) two possible strategies for deriving the cover.

\subsubsection{Negative set approximation}

This strategy assumes that the approximation is found from the STG-unfolding segment not only for $\mathcal{C}_{\mathcal{P}}^{*}$, covering the states in the $\mathcal{P}$-set, but also for $\mathcal{C}_{\mathcal{N}}^{*}$ which covers the states 
in the $\mathcal{N}$-set. Thus the partitioning of the segment for $\mathcal{C}_{\mathcal{N}}$ is also required. Assume that the approximations $\mathcal{C}_{\mathcal{P}}^{*}$ and $\mathcal{C}_{\mathcal{N}}^{*}$ were constructed so that all states in $\mathcal{P}$-set and $\mathcal{N}$-set, respectively, are covered. By making sure that $\mathcal{C}_{\mathcal{P}}^{*} \cdot \mathcal{C}_{\mathcal{N}}^{*}=\emptyset$ we guarantee that no state from $\mathcal{N}$-set is covered by $\mathcal{C}_{\mathcal{P}}^{*}$. However, if the intersection of approximations $\mathcal{C}_{\mathcal{P}}^{*}$ and $\mathcal{C}_{\mathcal{N}}^{*}$ is not empty, then the cover approximations must be refined, i.e. produce a new cover which covers less number of combinations. After full refinement, it must produce exact covers for $\mathcal{P}$-set and $\mathcal{N}$-set. If the refinement procedure terminates with fully refined covers but their intersection is still nonempty, then this STG does not satisfy the CSC condition.

This strategy finds approximations $\mathcal{C}_{\mathcal{P}}^{*}$ and $\mathcal{C}_{\mathcal{N}}^{*}$ which cover the required sets of states and partition the set of combinations which are allowed to be covered by them into two disjoint sets. It, therefore, produces pessimistic covers as it does not allow the combinations from the DC-set to be shared by both covers. The pseudo-code of the procedure implementing this strategy is shown in Figure 6(a).

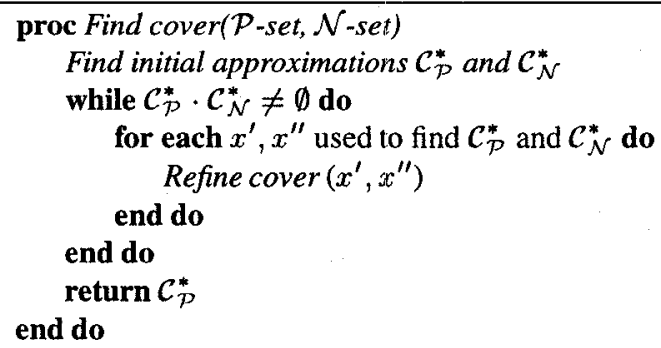

(a)

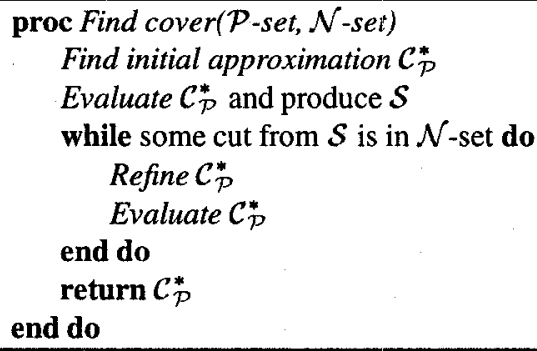

(b)

\section{Figure 6. Procedures for two cover deriving strategies}

\subsubsection{Positive set cover evaluation}

This strategy assumes that only the approximation $\mathcal{C}_{\mathcal{P}}^{*}$ covering the $\mathcal{P}$-set is found in the STG-unfolding segment. The cover $\mathcal{C}_{\mathcal{P}}^{*}$ is then evaluated by finding where in the STGunfolding segment it becomes TRUE. The evaluation finds a set of slices $\mathcal{S}$ which represent all states in which the cover becomes TRUE. If no cut in $\mathcal{S}$ represents a states from the $\mathcal{N}$-set, then the cover satisfies the correctness criterion. The pseudo-code of the procedure for this strategy is shown in Figure 6(b). Otherwise, approximation $\mathcal{C}_{\mathcal{P}}^{*}$ is refined until no cut represents a state from the $\mathcal{N}$-set. When $\mathcal{C}_{\mathcal{P}}^{*}$ is fully refined but such a cut still exists, the refining procedure aborts and reports the CSC violation.

The evaluation of the cover is done for each cube of the cover. It may use a branch-and-bound algorithm which splits a slice (the whole segment in the beginning) into subslices according to the literals present in each cube.

In this paper we concentrate on the first approximation strategy.

\subsection{Cover approximation}

The slices in the STG-unfolding segment represent two types of states: those where a signal transition $* a_{i}$ is excited $\left(G E R\left(* a_{i}\right)\right)$ and those in which the signal $a_{i}$ is stable $\left(G Q R\left(* a_{i}\right)\right)$. At the STG-unfolding level, we have instances of places and transitions. We can construct our slice cover approximations by means of the cover approximation for the place and transition instances which belong to the slice. The cover approximations can be divided into those for place instances $p^{\prime}$, representing the cuts containing these instances, and for transition instances $t^{\prime}$, representing the cuts enabling $t^{\prime}$. Obviously, it is possible to find an exact cover for an instance $x^{\prime}$ which will only cover cuts including $p^{\prime}$ or enabling $t^{\prime}$.

\subsubsection{Cover approximation for transition instance}

Consider, first, obtaining cover for a transition instance $* a_{i}^{\prime}$ in the STG-unfolding segment. All cuts in which $* a_{i}^{\prime}$ is excited are represented in its excitation slice $\mathcal{S}_{e}\left(* a_{i}^{\prime}\right)$. Any cut inside $\mathcal{S}_{e}\left(* a_{i}^{\prime}\right)$, reachable from the minimal excitation cut $C_{e}^{m i n}\left(* a_{i}^{\prime}\right)$, can only be reached by firing transitions which are concurrent to $* a_{i}^{\prime}$. If a signal instance $* a_{j}^{\prime}$ is concurrent to $* a_{i}^{\prime}$, then the value of its corresponding element in the binary code may take values of both " 0 " and " 1 ". The transition instance cover approximation $\mathcal{C}_{e}^{*}\left(a_{i}^{f}\right)$ is found from the binary code $\xi$ assigned to the cut $c_{e}^{m i n}\left(* a_{i}^{\prime}\right)$. The literals corresponding to the signals whose instances belong to $\mathcal{S}_{e}\left(* a_{i}^{\prime}\right)$ and are concurrent to $* a_{i}^{\prime}$ are substituted by "-." (don't care). Approximation reduces the number of literals in cover $\mathcal{C}_{e}^{*}\left(* a_{i}^{\prime}\right)$ and increases the number of combinations covered by $\mathcal{C}_{e}^{*}\left(* a_{i}^{\prime}\right)$.

Example. Consider the cover approximation for $+d^{\prime}$ in Figure $7(a)$. The binary code of its minimal excitation cut $c_{e}^{\text {min }}\left(+d^{\prime}\right)=\left(p_{2}^{\prime}, p_{3}^{\prime}, p_{4}^{\prime}\right)$ is found from the binary code of its local configuration as $\xi=\{1000000\}$ (the order of signals is abcdefg). There are four signals $\{b, c, e, f\}$ whose instances belong to the slice and are concurrent 


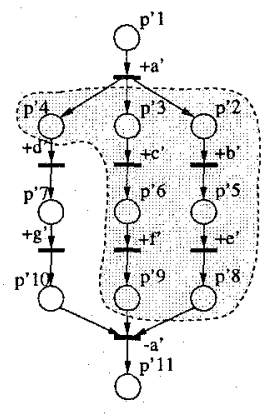

(a)

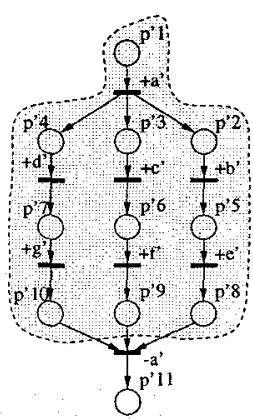

(b)

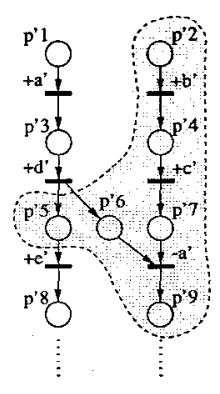

(c)
Figure 7. Illustration of cover approximation and refinement.

to $+d^{\prime}$. Thus the cover approximation for $+d^{\prime}$ will be $\mathcal{C}_{e}^{*}\left(+d^{\prime}\right)=\{1--0--0\}=a \bar{d} \bar{g}$.

Property 5.1 For any STG satisfying the general correctness criteria the cover approximation $\mathcal{C}_{e}^{*}\left(+a_{i}^{\prime}\right)\left(\mathcal{C}_{e}^{*}\left(-a_{i}^{\prime}\right)\right)$ obtained from its STG-unfolding segment of an STG always has the value of the element corresponding to $a_{i}$ set to " 0 " ("1").

\subsubsection{Cover approximation for place instance}

The place instance cover approximation $\mathcal{C}_{m}^{*}\left(p^{\prime}\right)$ for each $p^{\prime}$ is obtained from the binary code $\xi_{\left[t_{k}^{\prime}\right]}$ assigned to its preceding transition. Similar to transition instance cover approximation, any marking at which $p^{\prime}$ is marked can only be reached by firing transitions concurrent to $p^{\prime}$. The literals corresponding to signals whose instances belong to $\mathcal{S}$ and are concurrent to $p^{\prime}$ are replaced by "-.".

A place cover approximation must not cover markings outside the slice $\mathcal{S}$. The set of concurrent transitions is chosen so that it never leads to a cut which is outside the bounds of $\mathcal{S}$, i.e. its min-cut and the set of max-cuts. There may be several max-cuts. Thus for a place belonging to a max-cut, there may be several possibilities to choose the set of concurrent transitions. In this case, the place cover approximation is found as the union of all such possibilities: $\mathcal{C}_{m}^{*}\left(p_{l}^{\prime}\right)=\sum \mathcal{C}_{t_{k}^{\prime}}^{*}\left(p_{l}^{\prime}\right)$, where $t_{k}^{\prime}$ is the excluded concurrent transition.

Example. Consider approximation of the cover for places belonging to $\mathcal{S}\left(+a^{\prime}\right)=\left\langle\left(p_{1}^{\prime}\right),\left\{\left(p_{7}^{\prime}, p_{8}^{\prime}, p_{9}^{\prime}\right),\left(p_{6}^{\prime}, p_{8}^{\prime}, p_{10}^{\prime}\right)\right.\right.$, $\left.\left.\left(p_{5}^{\prime}, p_{9}^{\prime}, p_{10}^{\prime}\right)\right\}\right\rangle$ in the example shown in Figure $7(b)$. The approximation cover for $p_{4}^{\prime}$ is found from $\xi_{\left\lceil+a^{\prime}\right\rceil}$ as $\mathcal{C}_{m}^{*}\left(p_{4}^{\prime}\right)=$ $\{1--0--0\}=a \bar{d} \bar{g}$. Place $p_{10}^{\prime}$, on the other hand, belongs to at least one max-cut. Thus its approximation cover is found using two approximations corresponding to two different sets of concurrent transitions: $\mathcal{C}_{m}^{*}\left(p_{10}^{\prime}\right)=$ $\mathcal{C}_{f^{\prime}}^{*}\left(p_{10}^{\prime}\right) \quad\left(\right.$ for $\left.\quad\left\{b^{\prime}, c^{\prime}, e^{\prime}\right\}\right)+\mathcal{C}_{e^{\prime}}^{*}\left(p_{10}^{\prime}\right) \quad\left(\right.$ for $\left.\quad\left\{b^{\prime}, c^{\prime}, f^{\prime}\right\}\right)$ $=\{1--1-01\} \cup\{1--10-1\}=a d \vec{f} g+$ ade $g$.
Property 5.2 The cover approximation $\mathcal{C}_{m}^{*}\left(p^{\prime}\right)$ obtained from an on-set (off-set) slice $\mathcal{S}_{O n}\left(+a_{i}^{\prime}\right)\left(\mathcal{S}_{O f f}\left(+a_{i}^{\prime}\right)\right)$ in the STG-unfolding segment of an STG satisfying the general correctness criteria always has the value of the element corresponding to $a_{i}$ set to " 1 " (" 0 ").

\subsubsection{Finding $\mathcal{P}$-set and $\mathcal{N}$-set approximations}

Consider finding $\mathcal{P}$-set and $\mathcal{N}$-set approximation covers for each implementation architecture.

ACGpS implementation After the On- and Off-set partitioning w.r.t signal $a_{i}$ was found, the slices $\mathcal{S}_{O n}\left(+a_{i}^{\prime}\right)$ and $\mathcal{S}_{O n}\left(-a_{i}^{\prime}\right)$ represent the cuts starting from those enabling each instance $* a_{i}^{\prime}$ until (but not including) the cuts enabling next instances of $* a_{i}^{\prime}$. To approximate the cuts enabling $* a_{i}^{\prime}$ we use the transition instance approximation cover $\mathcal{C}_{e}\left(* a_{i}^{\prime}\right)$. To find the approximation for the rest of cuts in $\mathcal{S}_{O n}\left(+a_{i}^{\prime}\right)$ and $\mathcal{S}_{O n}\left(-a_{i}^{\prime}\right)$ we use a set of place instances which belong to $\mathcal{S}_{O n}\left(+a_{i}^{\prime}\right)$ and $\mathcal{S}_{O n}\left(-a_{i}^{\prime}\right)$, respectively.

For simplicity we only consider the on-set cover. The off-set cover approximation is found by using the instances of $-a_{i}$.

A cover approximation for a particular place $p_{l}^{\prime}$ will cover all states at which $p_{l}^{\prime}$ is marked with any other concurrent place $p_{j}^{\prime}$. Therefore, only mutually non-concurrent subset of places belonging to $\mathcal{S}_{O n}\left(+a_{i}^{\prime}\right)$ can be considered. A set of such places is found in the STG-unfolding segment.

Definition 5.1 A set of places $P_{a}^{\prime}$ belonging to the slice $\mathcal{S}_{O n}\left(+a_{i}^{\prime}\right)$ is called an approximation set of the slice $\mathcal{S}_{O n}\left(+a_{i}^{\prime}\right)$ iff $\forall p_{k}^{\prime} \in P_{a}^{\prime}: \quad+a_{i}^{\prime} \prec p_{k}^{\prime}$ and $\forall p_{k}^{\prime}, p_{l}^{\prime} \in P_{a}^{\prime}$ : $p_{k}^{\prime} \bigwedge p_{l}^{\prime}$.

The approximation set for a slice is a "skeleton" of places for this slice which are either sequential or in conflict with each other. Any cut encapsulated by $\mathcal{S}_{O_{n}}\left(+a_{i}^{\prime}\right)$ will contain a place from its approximation set.

The on-set cover approximation is found from $\mathcal{S}_{O n}\left(+a_{i}^{\prime}\right)$ as

$$
\mathcal{C}_{O n}^{*}\left(+a_{i}^{\prime}\right)=\mathcal{C}_{e}^{*}\left(+a_{i}^{\prime}\right)+\sum \mathcal{C}_{m}^{*}\left(p_{l}^{\prime}\right), p_{l}^{\prime} \in P_{a}^{\prime}
$$

and the whole on-set cover is found as:

$$
\mathcal{C}_{O n}^{*}\left(a_{i}\right)=\sum \mathcal{C}_{O n}^{*}\left(+a_{i}^{\prime}\right)
$$

Proposition 5.1 For any STG satisfying the general correctness criteria, the on-set cover approximation $\mathcal{C}_{O n}^{*}\left(a_{i}\right)$, obtained from its STG-unfolding segment, covers all reachable states in $O n\left(a_{i}\right)$. 
ACGpEF and ACGpER implementations Let us consider only calculation of the set function. For the reset function the instances of $-a_{i}$ are used. The $\mathcal{P}$-set and $\mathcal{N}$-set in these architectures are GER $\left(+a_{i}\right)$ and the rest of reachable states, respectively. In the discussion above, for the ACGpS implementation, we used two separate approximations for states in which $+a_{i}$ is excited and in which $a_{i}$ is stable. Thus the $\mathcal{P}$-set and $\mathcal{N}$-set cover approximations can be found by obtaining the On- and Off-set partitioning of the STG-unfolding segment.

The set cover approximation, covering the $\mathcal{P}$-set, is then found from the approximations of the excitation slices as:

$$
\mathcal{C}_{S}^{*}\left(a_{i}\right)=\sum \mathcal{C}_{e}^{*}\left(+a_{i}^{\prime}\right)
$$

Proposition 5.2 For any STG satisfying the general correctness criteria, the set cover approximation $\mathcal{C}_{S}^{*}\left(a_{i}\right)$, obtained from its STG-unfolding segment, covers all reachable states where $+a_{i}$ is excited.

The cover approximation for the $\mathcal{N}$-set is found as:

$$
\mathcal{C}_{\mathcal{N}}^{*}=\sum \mathcal{C}_{m}^{*}\left(p_{l}^{\prime}\right)+\sum \mathcal{C}_{e}^{*}\left(-a_{i}^{\prime}\right)+\sum \mathcal{C}_{m}^{*}\left(p_{k}^{\prime}\right)
$$

where $p_{l}^{\prime}$ and $p_{k}^{\prime}$ belong to the approximation sets found for On- and Off-set partitioning, respectively, as in the previous architecture.

Proposition 5.3 Let the $\mathcal{P}$-set and the $\mathcal{N}$-set be chosen according to the implementation architecture and the cover approximations $\mathcal{C}_{\mathcal{P}}^{*}\left(a_{i}\right)$ and $\mathcal{C}_{\mathcal{N}}^{*}\left(a_{i}\right)$ be calculated from the segment for a CSC-compliant STG in the above way. Then the Negative set approximation procedure given in Figure 6(a) produces correct covers for the chosen implementation architecture iff (1) the refinement procedure restores the exact covers for $\mathcal{P}$-set and the $\mathcal{N}$-set in a finite number of iterations and (2) every refinement step produces a cover which covers less number of combinations.

Note that only the intersection between cover approximations for excitation slices and the places of the Off-set partitioning needs to be checked when the set function is found for the implementation in the ACGpEF or ACGpER architectures. This reduces the number of cover intersections to be checked and, hence, reduces the synthesis time.

\subsection{Cover refinement}

The purpose of the refining procedure is to restore some of the relations between concurrent transitions belonging to a slice $\mathcal{S}$ which were ignored during the approximation. If the intersection of two covers is non-empty, then a set of offending signal $\mathrm{Sig}$ is found from a pair of intersecting cubes. These are the signals whose corresponding literals are missing from a particular cube of the cover.
The refinement of cover for element $x^{\prime}$ is based on finding the refinement set for $x^{\prime}$ w.r.t. an offending signal $a_{j}$.

Definition 5.2 A set of places $P_{r}^{\prime}$ belonging to a slice $\mathcal{S}$ is called the refining set of $x^{\prime}$ w.r.t. $a_{j} \in S i g$ iff it satisfies the following: $\forall p_{k}^{\prime} \in P_{r}^{\prime}: x^{\prime} \| p_{k}^{\prime}, \forall p_{k}^{\prime}, p_{l}^{\prime} \in P_{r}^{\prime}: p_{k}^{\prime} \quad \chi \mid p_{l}^{\prime}$ and $\forall * a_{j}^{\prime} \in \mathcal{S}:\left(* a_{j}^{\prime}\right) \bullet \cap P_{r}^{\prime} \neq \emptyset$.

In other words, the refining set is a set of mutually nonconcurrent places belonging to $\mathcal{S}$ which are pair-wise concurrent with $x^{\prime}$. The condition on the inclusion of the successors of each $* a_{j}^{\prime}$ into $P_{r}^{\prime}$ is constituted by the progress requirement of the refinement procedure. Thus at least one signal will be refined at each iteration.

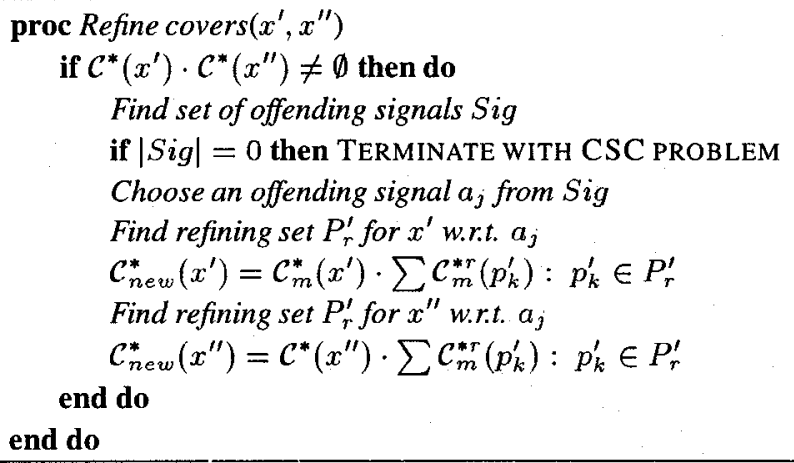

Figure 8. Procedure for refining cover approximations.

A refined cover $\mathcal{C}_{\text {new }}^{*}\left(x^{\prime}\right)$ is obtained from the old approximation as: $\mathcal{C}_{n e w}^{*}\left(x^{\prime}\right)=\mathcal{C}^{*}\left(x^{\prime}\right) \cdot \sum \mathcal{C}_{m}^{* r}\left(p_{k}^{\prime}\right), p_{k}^{\prime} \in P_{r}^{\prime}$. The cover $\mathcal{C}_{m}^{* r}\left(p_{k}^{\prime}\right)$ is a restricted cover approximation for $p_{k}^{\prime}$, where only those literals are set to "_" whose instances, concurrent to $p_{k}^{\prime}$, belong to $\mathcal{S}$ and are successors of $x^{\prime}$. Unlike the ordinary place instance cover, $\mathcal{C}_{m}^{* r}\left(p_{k}^{\prime}\right)$ uses the restricted set of transitions to exclude (from the set of concurrent to $p_{k}^{\prime}$ ) those who have already fired while reaching $x^{\prime}$.

The pseudo-code of the algorithm for cover refinement is shown in Figure 8.

Proposition 5.4 The synthesis procedure given in Figure 6(a) is finite for any implementation architecture.

Proof: [Sketch] Since each step refines the value of at least one variable and the set of signals is finite, the refinement procedure will terminate after at most $|A| \times\left|P_{a}^{\prime}\right|$ iterations. If at the end of refinement any two covers intersect, then the STG has CSC problem which will be reported.

Proposition 5.5 The fully refined (in the worst case) cover of $x^{\prime}$ in the STG-unfolding segment built for a CSCcompliant STG covers only states corresponding to the cuts 
which are encapsulated by $\mathcal{S}$ and covered by the exact cover for $x^{\prime}$.

Proof: [Sketch] The approximated cover for $x^{\prime}$ represents partial markings: those where input places of $x^{\prime}$, if $x^{\prime}$ is a transition, and those where $x^{\prime}$ is marked, if $x^{\prime}$ is a place. At each step the refinement procedure restores the marking component of reachable states represented by $\mathcal{S}$. It finds a set of places which can be marked together with each already partially restored marking. The cover function is changed to reflect the fact that partial markings now include the places found. Thus in the end, when the procedure terminates, the covers correspond to fully restored markings and cover only states with these marking components.

Corollary 5.1 The fully refined cover for an excitation slice is equal to the exact cover of this slice.

Corollary 5.2 The fully refined cover for an on- (off-) slice is equal to the exact cover of this slice.

From the above corollaries it follows that Proposition 5.3 holds. Therefore, our chosen strategy produces correct covers for any CSC-compliant STG. Moreover the refinement procedure preserves covering of the $\mathcal{P}$-set. Therefore, after each iteration step a newly obtained cover also covers $\mathcal{P}$-set. Hence, if after some iteration the new $\mathcal{P}$-set and $\mathcal{N}$ set covers have empty intersection, then they will satisfy the implementation specific correctness criterion, set out in Definitions 2.1-2.3 for each implementation architecture.

Example. Consider a fragment of STG-unfolding segment shown in Figure 7(c). Suppose that on-set cover approximation $\mathcal{C}_{O n}^{*}$, found with approximation set $P_{a}^{\prime}=$ $\left\{p_{1}^{\prime}, p_{3}^{\prime}, p_{5}^{\prime}, p_{8}^{\prime}\right\}$, intersects with $\mathcal{C}_{O f f}^{*}$ for some signal. Suppose also that a cube $B=d \bar{e}$ which is an cover approximation of place $p_{5}^{\prime}$ causes this non-empty intersection. The set of offending signals is found as Sig $=\{a, b, c\}$. Let $a$ be the signal chosen for refinement. Its only instance which should be used in refinement is $-a^{\prime}$. A refinement set is chosen as $P_{r}^{\prime}=\left\{p_{2}^{\prime}, p_{4}^{\prime}, p_{7}^{\prime}, p_{9}^{\prime}\right\}$. Consider calculation of the restricted cover approximation for $p_{2}^{\prime}$. The only instances which can be used in approximation is $+e^{\prime}$ as other concurrent instances, $+a^{\prime}$ and $+d^{\prime}$, precede $p_{5}^{\prime}$. Thus $\mathcal{C}_{m}^{* r}\left(p_{2}^{\prime}\right)=\{1001-\}$ (the order of signals is abcde). Similar, cover approximations are found for other places in $P_{r}^{\prime}$. The refined cover approximation is thus found as: $\mathcal{C}_{\text {new }}^{*}\left(p_{5}^{\prime}\right)=\{---10\} \cap[\{1001-\} \cup\{1101-\} \cup$ $\{1111-\} \cup\{0111-\}]=a \bar{c} d \bar{e}+b c d \bar{e}$. The resulting cover is an exact cover of for place $p_{5}^{\prime}$.

\section{Experimental results}

We implemented the method introduced in this paper on the basis of the already existing (STG verification) tool

\begin{tabular}{|c|c|c|c|c|c|c|}
\hline \multirow[t]{2}{*}{ Benctimark } & \multirow[t]{2}{*}{ Sigs } & \multicolumn{2}{|c|}{ PUNT ACGSS } & \multicolumn{3}{|c|}{ Other tools } \\
\hline & & TotTim & Licm & Petrify & & LitCot \\
\hline imec-master-read.csc & 18 & 7.00 & 83 & 125.66 & 630.52 & 69 \\
\hline Bowick aso & 7 & 0.97 & 17 & 1,44 & 0.51 & 20117 \\
\hline nowick & 6 & 0.57 & 15 & 1.10 & 0.23 & 14 \\
\hline par-4.csc & 14 & 3.63 & 36 & 12,31 & 168.55 & 36 \\
\hline sis-master-read.csc & 14 & 5.78 & 48 & 27.09 & 130.66 & 48 \\
\hline tsomSIBRK & 25 & 42.70 & 72 & 299.90 & 141.51 & $\mathbf{7 2}$ \\
\hline pn_stg_example & 6 & 1.77 & 19 & 4.20 & 6.84 & 19 \\
\hline forever_onder & 8 & 1.46 & 20 & 5.24 & 8.81 & 16 \\
\hline alloc-ortbound & 9 & 0.85 & 16 & 1.75 & 1.53 & 16 \\
\hline mp-forward-pkt & 20 & 0.83 & 17 & 1.50 & 0.22 & 17 \\
\hline nak-pa & 10 & 0.96 & 20 & 2.28 & 0.29 & 20 \\
\hline pe-send-ifc & 17 & 2.53 & 68 & 19.50 & 1.16 & $75 / 72$ \\
\hline ram-read-shuf & 11 & 1.08 & 25 & 3.28 & 0.26 & 22 \\
\hline rCY-setup & 5 & 0.25 & 8 & 0.72 & 0.14 & 8 \\
\hline sbuf-ram-write & 12 & 1.48 & 23 & 4.04 & 0.38 & 23 \\
\hline sbuf-read-ct.old & 8 & 0.86 & 15 & 1.29 & 0.19 & 15 \\
\hline sbuf-read-ctl & 8 & 0.71 & 15 & 0.99 & 0.16 & 15 \\
\hline shuf-send-ell & 8 & 0.88 & 19 & 1.95 & 0.21 & 19 \\
\hline sbuf-send-pk12 & 9 & 0.99 & 19 & 2.16 & 0.23 & 19 \\
\hline & 9 & 1.07 & 31 & 3.43 & 0.26 & 31 \\
\hline sendr-done & 4 & 0.23 & 6 & 0.33 & 0.14 & 6 \\
\hline Total & 228 & 146.78 & 592 & 520.16 & 1092.77 & $580 / 574$ \\
\hline
\end{tabular}

Table 1. Experimental results

"PUNT", which constructs an STG-unfolding segment. The Negative set approximation strategy requires On- and Off-set partitioning for each of our implementation architectures. Therefore, we chose ACGpS as an indicator of the method's performance. While testing the new method we pursued two objectives:

- To demonstrate the practicality of our approach on a set of moderate sized examples.

- To illustrate the increased feasibility of synthesis process using our approach.

\subsection{Practicality}

To demonstrate the practicality of our approach we chose a set of publicly available benchmarks. All STGs in this set of benchmarks have low number of signals (max. 25). Table 1 presents total time spent (in seconds) on synthesis of speed-independent circuits from their STG specifications in the ACGpS architecture ("PUNT ACGpS"). On average, about $1 \%$ of this time was spent on building the STGunfolding segment and about $15 \%$ was spent on Espresso minimisation. For comparison, the same set of benchmarks was synthesised using Petrify and SIS $[3,15]$. Their total timings are grouped in the column "Other tools".

To illustrate the practicality of our method we used literal count (columns "LitCnt"). The literal count shows the total number of literals used in all the cubes of logic functions implementing all signals. As it can be observed, our synthesis technique produces implementations comparable with those produced by other tools. The timing results show that it compares favourably to Petrify and becomes increasingly better than SIS with the growth of the signal count. These results show that for small sized benchmarks, the overheads of constructing and traversing the STG-unfolding segment may outweigh the time spent on constructing a small reachability graph with an efficient implementation. Slightly worse literal count for some benchmarks is attributed to the fact that the DC-set is partitioned due to a stricter cover cor- 


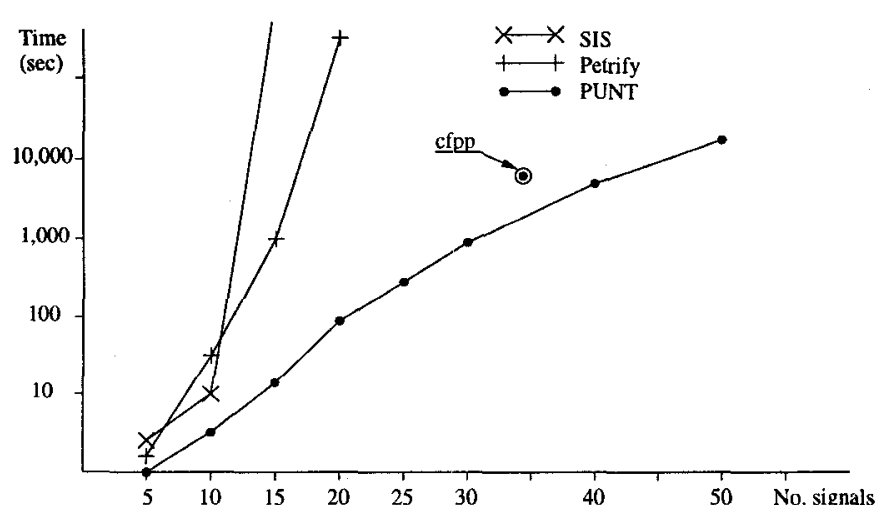

Figure 9. Experimental results for Muller pipeline.

rectness condition.

\subsection{Feasibility}

To illustrate feasibility we chose the Muller pipeline benchmark. The graph interpretation of the results is shown in Figure 9. As can be observed, the existing methods cannot cope well with the growing size of the specification, either running out of memory or taking prohibitively long time. Double exponential dependency of SIS and Petrify is attributed to (1) exponential explosion of the state space, and (2) exact cover calculation methods. In addition, we synthesised a Counterflow pipeline specification [17] which has 34 signals. From the existing tools, only Petrify was able to synthesise it but it took more than 24 hours. At the same time PUNT was able to synthesise it in under 2 hours, thus giving an order of magnitude gain in speed. This result is shown on the graph as a circled dot.

\section{Conclusions}

In this paper we presented a new method for synthesis of speed independent circuits. Our approach is based on the STG-unfolding segment. It uses the segment as a model from which an implementation is obtained. As the size of the STG-unfolding segment is often smaller than that of the SG, it is possible to synthesise larger circuits. In addition, due to the smaller size of the semantic model, the implementation can be achieved faster on a number of moderate sized examples.

We demonstrated applicability of our method on an existing set of benchmarks. Our method can be used with the widest class of STG specifications. Future development of this method can be directed into exploring heuristics for the refinement procedure.

\section{References}

[1] P. Beerel. CAD Tools for the Synthesis, Verification and Testability of Robust Asynchronous Circuits. PhD thesis, Stanford University, 1994.

[2] T. Chu. Synthesis of Self-Timed VLSI Circuits from Graphtheoretic Specifications. PhD thesis, MIT, 1987.

[3] J. Cortadella et. al. Petrify: a tool for manipulating concurrent specifications and synthesis of asynchronous controllers. In Proc. of the 11th Conf. Design of Integrated Circuits and Systems, pages 205-210, Barcelona, Spain, Nov. 1996.

[4] J. Esparza, S. Römer, and W. Vogler. An inprovement of mcmillan's unfolding algorithm. Technical Report TUM-I9599, Insitute Für Informatik, Technische Universität München, 1995.

[5] M. Kishinevsky, A. Kondratyev, A. Taubin, and V. Varshavsky. Concurrent Hardware: The Theory and Practice of Self-Timed Design. John Wiley and Sons, London, 1993.

[6] A. Kondratyev, M. Kishinevsky, B. Lin, P. Vanbekbergen, and A. Yakovlev. Basic gate implementation of speedindependent circuits. In Proceedings of Design Automation Conference, pages 56-62, June 1994.

[7] A. Kondratyev and A. Taubin. On verification of the speedindependent circuits by STG unfoldings. In ASYNC'94, Salt Lake City, Utah, USA, November 1994.

[8] K. McMillan. Symbolic Model Checking. Kluwer Academic Publishers, Boston, 1993.

[9] T. Miyamoto and S. Kumagai. An efficient algorithm for deriving logic functions of asynchronous circuits. In Proc. of ASYNC'96, pages 30-35, Aizu-Wakamatsu, Fukushima, Japan, March 1996.

[10] E. Pastor, J. Cortadella, A. Kondratyev, and O. Roig. Structural methods for the synthesis of speed-independent circuits. In Proc. European Design and Test Conference, pages 340-347, Paris(France), March 1996.

[11] W. Reisig. Petri Nets, An Introduction. Springer-Verlag, 1985.

[12] L. Rosenblum and A. Yakovlev. Signal graphs: from selftimed to timed ones. In Proceedings of International Workshop on Timed Petri Nets, Torino, Italy, July 1985, pages 199-207.

[13] A. Semenov and A. Yakovlev. Event-based framework for verification of high-level models of asynchronous circuits. Technical Report 487, University of Newcastle upon Tyne, 1994.

[14] A. Semenov, A. Yakovlev, E. Pastor, M. P. na, and J. Cortadella. Synthesis of speed independent circuits from STGunfolding segment. Technical report, University of Newcastle upon Tyne, 1996.

[15] E. Sentovich et. al. SIS: A system for sequential circuit synthesis. Memorandum No. UCB/ERL M92/41, University of California, Berkeley, 1992.

[16] P. Siegel and G. D. Micheli. Decomposition methods for library binding of speed-independent asynchronous designs. In Proceedings of the International Conference on Computer-Aided Design, pages 558-565, 1994.

[17] A. Yakovlev. Designing control logic for counterflow pipeline processor using Petri nets. Technical Report 522, University of Newcastle upon Tyne, 1995.

[18] C. Ykman-Couvreur, B. Lin, and H. DeMan. ASSASSIN: A synthesis system for asynchronous control circuits. Reference manual, IMEC, 1995. 